Published in Gérontologie et société, 2020, vol. 42 no. 161, pp. 87-99 which should be cited to refer to this work.

DOI : http://doi.org/10.3917/gs1.161.0087

\title{
Parents d'adultes avec une déficience intellectuelle : un engagement pour la vie
}

\section{Adeline PAIGNON}

Professeure en éducation interprofessionnelle, Docteure en Psychologie, Haute école de santé Genève, HES-SO Haute école spécialisée de Suisse occidentale et Centre interprofessionnel de simulation Genève

\section{Séverine LAVERRIÈRE}

Infirmière, Conseillère aux études, Haute école de santé Genève, HES-SO Haute école spécialisée de Suisse occidentale

\section{Séverine LALIVE D’ÉPINAY RAEMY}

Maître d'enseignement, Infirmière en soins infirmiers généraux, Haute école de santé

Genève, HES-SO Haute école spécialisée de Suisse occidentale

\section{Françoise CINTER}

Professeure en soins infirmiers, Infirmière en soins infirmiers généraux, Master en Sciences de l'éducation, Haute école de santé Genève, HES-SO Haute école spécialisée de Suisse occidentale

e-mail auteure : adeline.paignon@hesge.ch 
Résumé - Face à l'allongement de l'espérance de vie dans nos sociétés actuelles, les instances socio-éducatives et de santé s'interrogent sur le devenir des personnes en situation de handicap. En Suisse, le vieillissement conjoint des parents et de leurs enfants en situation de handicap avec une déficience intellectuelle préoccupe ces différentes instances. Qu'elles vivent en internat ou en externat, le vieillissement de ces personnes confronte les parents, les professionnels, les institutions, le système socio-sanitaire à la prise en compte de nouvelles dimensions dans l'accompagnement, notamment celle du suivi de santé. C'est dans ce contexte que nous questionnons l'engagement des parents dans le suivi de santé de leur fils/fille et de ce qu'il implique pour chacun des acteurs du réseau d'accompagnement.

Mots clés -

parents, suivi de santé, handicap, déficience intellectuelle, engagement

\section{Abstract - The parents of adults with intellectual disabilities: A commitment for life}

Faced with the increase in life expectancy in our current societies, socioeducational and health authorities are examining the future of people with disabilities. In Switzerland, the simultaneous aging of parents and their children with intellectual disabilities is a concern for these specific authorities. Whether they live in supported residential accommodation or go to day centers, the aging of people with intellectual disabilities confronts parents, professionals, institutions, and the social and healthcare system with increasing needs of support, particularly regarding health monitoring. It is in this context that we examine the commitment of parents in the health monitoring of their son/daughter. We also consider the impact on each of the actors in the social and healthcare network.

Keywords - parents, health monitoring, disability, intellectual disability, commitment 


\section{Introduction}

En Suisse, en 2018, environ 1656000 personnes âgées de plus de 15 ans étaient en situation de handicap, dont la moitié avec un handicap mental (Office fédéral de la statistique, 2018). Une partie d'entre elles présentent une déficience intellectuelle qui réfère à " un niveau de fonctionnement cognitif global, significativement inférieur à la moyenne, accompagné d'une réduction des capacités d'adaptation aux exigences quotidiennes de l'environnement social » (Association francophone d'aide aux handicapés mentaux, 2013, p. 1). Cette situation de handicap engendre une organisation familiale spécifique qui se caractérise la plupart du temps par un engagement ${ }^{1}$ des parents ${ }^{2}$ auprès de leur enfant tout au long de la vie (Gardou, 2016) et qui se confronte dorénavant au vieillissement conjoint des parents et de leur enfant (Breitenbach, 2003). En Suisse, il s'avérait déjà qu'en 2010 environ $70 \%$ des parents accompagnant leur enfant avaient entre 54 et 76 ans et 16 \% plus de 77 ans (Jecker-Parvex et al., 2010). Les nouvelles problématiques rencontrées par les parents face à leur vieillissement et celui de leur enfant doivent donc être prises en compte pour éviter des ruptures dans leurs accompagnements (Berkman, 2006 ; Piazza et al., 2014). Dans ce contexte, nous nous intéressons plus particulièrement aux impacts de l'engagement des parents dans le suivi de santé de leur enfant sur leur propre vie et leur rôle dans le réseau d'accompagnement de leur fils ou fille devenu adulte.

\section{Un engagement inscrit dans le cadre légal en Suisse}

Dans la plupart des pays européens, les parents jouent un rôle important dans le parcours de vie de leur fils/fille en situation de handicap. Dès leur naissance, la plupart des parents sont présents pour guider leur enfant sur le chemin de la vie. Ils vont l'accompagner dans une société qui n'est pas forcément prête à l'accueillir. Entre la reconnaissance du handicap, sa prise en charge à domicile ou en institution, l'inclusion scolaire, couplées aux problèmes de santé associés ou non au handicap, les parents sont perpétuellement à l'affût des meilleures solutions pour garantir le bien-être et le développement des potentiels de leur fils/fille. Si ces parents restent, de différentes façons, présents dans la vie de leur enfant même à l'âge adulte, la place qu'ils occupent auprès de leur fils/fille

\footnotetext{
${ }^{1}$ L'engagement des parents étant entendu ici dans une double dimension. Celle de leurs implications dans les aspects concrets de la vie de leur enfant et celle de leurs implications pour le respect de la dignité et la défense des droits de leur enfant.

${ }^{2} \mathrm{Au}$ sens de père et/ou mère.
} 
avec une déficience intellectuelle, présente des caractéristiques singulières. Cette présence dans l'accompagnement perdure de façon significative bien souvent au-delà de la majorité de leur fils/fille. En Suisse, les personnes adultes avec une déficience intellectuelle, lorsqu'elles sont reconnues dans l'incapacité de discernement, sont protégées par une curatelle de portée générale dès leur majorité (Art. 398, Code civil suisse). Il s'agit d'une mesure de protection en faveur des personnes qui ont besoin d'aide. Généralement attribuée aux parents sur décision judiciaire par les autorités compétentes, cette curatelle leur confère la responsabilité des domaines de l'assistance personnelle, y compris la représentation dans le domaine médical (Art. 377-381, Code civil suisse), la gestion du patrimoine et les rapports juridiques avec les tiers. De facto, ces parents s'inscrivent dans une forme de parentalité sans fin, qui lie leur vie à celle de leur enfant devenu adulte pour lequel ils devront prendre des décisions jusqu'à ne plus pouvoir les assumer (Wicki, 2016). Si les parents délèguent parfois certains des aspects de la curatelle au profit d'un tiers désigné ou volontaire, ils conservent en général leur prérogative dans le suivi de santé de leur proche. En référence aux recommandations de l'Académie suisse des Sciences médicales (2008), nous entendons ici par suivi de santé, toutes les actions et mesures qui favorisent la prévention, le dépistage, le diagnostic médical et les thérapies usuelles adaptés à l'âge, au sexe de la personne comme pour l'ensemble de la population et les mesures spécifiquement adaptées à leur handicap. Cette représentation légale dans le domaine médical amène donc les parents à gérer le suivi de santé et implique qu'ils participent activement aux décisions de santé. Ils sont ainsi non seulement des acteurs du réseau familial, mais aussi du réseau socio-sanitaire qui accompagne leur enfant, et en deviennent un maillon indispensable et incontournable. Pourtant, ils sont peu reconnus dans ce rôle et pourraient être considérés comme des partenaires " cachés » du système d'accompagnement.

\section{Un engagement au long cours}

En Suisse, la prise en charge dans des institutions spécialisées reste souvent privilégiée pour les adultes avec une déficience intellectuelle (Jecker-Parvex et Breitenbach, 2012). Elle se présente sous deux formes : l'externat avec un retour à domicile journalier et l'internat avec un retour à domicile régulier (un week-end sur deux, par exemple). Bien que leur prise en charge soit assurée par des professionnels socio-éducatifs et de santé, il n'en demeure pas moins que les parents restent répondants légalement de la santé de leur fils/fille. Ce sont eux qui sont habilités à collaborer aux prises de décision concernant les soins et 
investigations proposées par les médecins. Eux qui gèrent et transmettent aux professionnels les suites des soins à prodiguer telles que la vérification de l'administration des médicaments et leur gestion. Outre la responsabilité légale qui leur incombe, ils disposent d'une connaissance approfondie des besoins de leur fils/fille acquise au cours de leur vie et ils pensent souvent que personne d'autre ne serait capable de les remplacer (Lamura, Döhner et Kofahl, 2008). Ainsi, comme le soulignent Bernard Azéma et Nathalie Martinez (2005), les parents jouent un rôle fondamental dans la prise en soin dès lors que nombre de problèmes de santé ne sont pas reconnus ou sont sous-estimés par les professionnels, voire totalement négligés par les dispositifs sanitaires et sociaux. Les études de différentes instances font d'ailleurs l'amer constat que l'accès aux soins est réduit pour les personnes quelle que soit leur situation de handicap (Office fédéral de la statistique, 2017 ; Organisation mondiale de la santé, 2011), et ce d'autant plus pour les personnes non communicantes (Unapei, 2013 ; Lalive d'Epinay Raemy et Paignon, 2019). Cet état de fait est régulièrement pointé du doigt par les parents. Ils se rendent bien souvent compte que les professionnels ne s'inquiètent que très peu face aux comportements inhabituels de leur fils/fille, alors que ces comportements expriment en réalité l'inconfort de leur enfant voire une douleur signalant un problème de santé plus aigu. Dans le même temps, ils sont préoccupés par les interpellations incessantes dont ils font l'objet de la part des professionnels d'accompagnement pour décrypter ces changements de comportements. Ils sont très souvent sollicités pour les interpréter et décider des suites à donner. Ils éprouvent alors un sentiment ambivalent à la fois d'impuissance ou de toutepuissance, face à l'inefficacité de la prise en charge qui renforce encore la nécessité de leur engagement et de leurs interventions. Ils sont ainsi très largement impliqués dans les aspects concrets du suivi de santé de leur fils/fille. Ils pallient souvent la désaffection des professionnels socio-éducatifs qui n'ont pas les moyens de réaliser ce suivi et qui n'ont pas été formés pour l'assurer. Ainsi, ce sont souvent les parents qui effectuent les démarches administratives et accompagnent leur proche chez le médecin, le dentiste ou à l'hôpital (Campéon et Le Bihan-Youinou, 2016), même lorsque celui-ci est en internat. Ils doivent aussi faire face aux imprévus et aux situations d'urgence pour lesquelles les professionnels d'accompagnement sont insuffisamment formés. Leur connaissance approfondie, l'expertise acquise dans les besoins de leur enfant devenu adulte, les soins et l'accompagnement prodigués depuis la naissance, les conduit à maîtriser les méandres du réseau socio- sanitaire et pouvoir s'y engager comme des acteurs incontournables (Carlsson et al., 2016). Ils ont appris à collaborer avec les professionnels de santé qui ont jalonné la vie de leur 
fils/fille dans la gestion des maladies, des accidents, des hospitalisations, et ils veillent continuellement à assurer le bien-être et la santé de leur proche. Dès lors, au-delà du rôle d'accompagnant sécurisant et détenteur de l'histoire médicale de leur enfant devenu adulte, les parents endossent celui de coordinateur des interventions et centralisent l'ensemble des informations de santé, en veillant à ce que son suivi soit adéquat (Seltzer et al., 2011). Ils deviennent le liant, l'interface entre leur enfant et le système socio-sanitaire, mais ce rôle peut devenir difficile à assumer à certaines périodes de leur vie, en particulier lorsqu'ils arrivent à un âge avancé.

\section{Un engagement aux impacts contrastés pour les parents}

Les études réalisées sur l'impact de l'engagement des parents auprès de leur fils/fille sur leur propre qualité de vie présentent des résultats hétérogènes. Cependant, force est de constater que l'engagement parental auprès d'un enfant en situation de handicap impacte leur qualité de vie. Cet engagement peut avoir des conséquences négatives sur la santé physique et psychique des parents. Cela se manifeste notamment par un niveau de stress élevé qui peut conduire à une diminution des capacités immunitaires, une augmentation des risques de maladies cardio-vasculaires, par exemple. Le stress ressenti en continu peut produire un déséquilibre émotionnel et altérer la capacité de faire face aux événements du quotidien. L'anxiété, les ruminations, les troubles de la mémoire et de la concentration peuvent alors émerger et rendre les parents plus vulnérables (Schneiderman, Ironson et Siegel, 2005). Leur vie sociale, conjugale, ou professionnelle peut aussi s'en trouver dégradée. Cela peut les amener à un isolement et au sentiment de ne plus être un membre de la société mais être seulement " un parent d'enfant en situation de handicap " reconnu comme tel par l'environnement social (Korff-Sausse, 2007 ; Scelles, 2006). À relever que les conséquences de cet engagement sont aussi liées à la période de vie de chacun. D'une façon générale, nous pouvons identifier trois périodes dans la littérature scientifique et dans les témoignages de parents. Ainsi, on distingue une première période, de l'annonce du diagnostic de handicap jusqu'à la fin de la petite enfance, pendant laquelle on observe une forte altération de la qualité de vie des parents (Siu et al., 2018). Ils éprouvent de la colère, ressentent de l'injustice, associées à un sentiment de fardeau important, et un stress élevé face à l'inconnu et aux incertitudes liées au devenir de leur enfant. Au cours de l'adolescence et pendant la transition à l'âge adulte - bien que cette deuxième période de vie induise un niveau de stress élevé, le sentiment de bien- 
être des parents n'est pas autant altéré que dans la période précédente (Pozo et Sarriá, 2015). Un certain équilibre familial et professionnel a pu se mettre en place et les parents ont appris à vivre avec cet enfant différent. Chez les parents plus âgés, la qualité de vie est reconnue comme amoindrie. Les nouvelles contraintes auxquelles ils sont soumis telles que la diminution de leur force physique, le déclin de leur propre santé et de nouvelles responsabilités comme de prendre soin de leurs propres parents ou de leur conjoint affectent leur vie quotidienne (Seltzer et al., 2011). Ces sollicitations supplémentaires vont impacter leur vie et potentiellement limiter leurs ressources pour faire face à des événements imprévus de la vie de leur fils/fille adulte (Cuskelly, 2006). Cela peut les amener à un conflit interne : comment continuer à s'occuper de leur enfant alors que leurs ressources internes s'amoindrissent ? De plus, la question récurrente du devenir de leur proche lorsqu'ils ne seront plus à même de s'en occuper et après leur disparition devient plus prégnante (Thomas, Hazif-Thomas et Clément, 2005).

Si les effets négatifs de l'engagement des parents auprès de leur enfant ont été largement documentés, il a aussi été montré qu'ils en retirent des bénéfices. Ainsi, quelle que soit la période de vie, de nombreux parents estiment que leur situation leur donne une grande satisfaction, que c'est une expérience riche et gratifiante (Lindblad, Rasmussen et Sandman, 2005) et une source de dépassement de soi (Ooi et al., 2016). L'expertise que les professionnels leur reconnaissent contribue à leur donner un sentiment de confiance dans leurs compétences et dans leur pouvoir d'agir (Waizbard-Bartov, Yehonatan-Schori et Golan, 2019). Ils ont acquis des capacités d'adaptation face aux imprévus qu'ils peuvent mobiliser dans le contexte familial, professionnel, ou associatif. II faut cependant prendre en considération que de nombreux facteurs interagissent entre eux et déterminent la façon dont l'engagement des parents va impacter leur vie. Les facteurs tels que les caractéristiques individuelles (âge des parents, type et degré de handicap de l'enfant), le fardeau ressenti, le soutien social et familial, les conditions socio- économiques, le contexte légal et sociétal du pays (droit des parents, ressources communautaires) sont effectivement considérés comme autant de dimensions déterminantes de la qualité de vie parentale.

La plupart des études mentionnées précédemment ne présentent que très peu de données récoltées auprès des parents seniors. Pourtant cette période de vie semble présenter des spécificités non seulement liées à l'âge des parents mais aussi à celui de leur fils/fille qui présente de nouvelles problématiques de santé complexes. Dans le cadre de la gestion de ces problématiques de santé, nous 
avons été interpellées par les propos de parents quant aux difficultés qu'ils rencontraient pour la prise en soin de leur enfant compte tenu de leur propre état de fatigue et de santé. Nous avons souhaité explorer avec eux ces difficultés et réfléchir ensemble aux mesures de soutien qui permettraient de les soulager dans cette période de vie.

\section{Comprendre et soutenir l'engagement des parents vieillissants : une étude à Genève}

Dans l'étude RéPA-Handi ${ }^{2}$, en cours à Genève, nous cherchons à identifier avec des parents de tous âges, les difficultés et les ressources qui leur sont nécessaires pour garantir le bien-être et le suivi de santé de leur proche (Cinter et Paignon, 2019). Nous nous interrogeons particulièrement sur l'impact de leur engagement relatif à chaque période de vie. L'objectif visé est d'évaluer avec des parents, la faisabilité et les modalités possibles d'un passage de relais entre parents et professionnels pour le suivi de santé de leur fils/fille avec une déficience intellectuelle.

Pour cela, nous invitons, par courrier et lors de séances d'informations, les parents curateurs, membres d'une association, intéressés par la thématique à participer à l'étude. Cette participation leur offre un espace de parole et de réflexion sur leur propre réalité et de se soutenir mutuellement (Deschamps, 2003). Notre étude comporte un volet quantitatif qui permet, entre autres de recueillir les profils sociodémographiques de la dyade parents/enfant, leurs vécus psycho-émotionnels et une description de leurs activités et de leurs relations aux professionnels concernant le suivi de santé de leur fils/fille. Un second volet qualitatif est proposé aux parents qui souhaitent partager leurs expériences en groupe lors de séances de focus group. Ces séances permettent d'objectiver, tant pour l'équipe de recherche que pour les parents-participants eux-mêmes, un périmètre d'actions et de mesures commun au suivi de santé de leurs fils/filles adultes quelle que soit leur situation de handicap subséquente à leur déficience intellectuelle. Enfin, de recueillir leurs attentes quant à la faisabilité d'un passage de relais progressif pour maintenir le suivi de santé de leur fils/fille dans le cadre légal existant.

\footnotetext{
2 «Parents sentinelles de la santé de leurs enfants en situation de handicap avec déficience intellectuelle : élaboration d'une modalité de répit innovante à une parentalité sans fin. " Ce projet en collaboration avec l'association insieme Genève (Association de parents et d'amis de personnes mentalement handicapées) s'inscrit dans une collaboration plus large entre les domaines Santé des hautes écoles suisses et vise à créer un centre.
} 
Dans l'ensemble des données récoltées au cours de l'étude, les parents expriment clairement leur envie de poursuivre leur implication dans le suivi de santé de leur fils/fille, ce qui donne du sens à leur vie et les maintient actifs dans le réseau social. Cependant, ils relèvent qu'avec leur avancée en âge, ils éprouvent une forme d'épuisement se traduisant par une importante fatigue physique et l'émergence de nouveaux problèmes de santé. Pourtant, comme le montrent nos résultats, ils n'utilisent que très rarement les solutions de répit proposées aux proches aidants. Selon eux, ces dispositifs s'adressent aux proches aidants et ils ne se reconnaissent pas dans cette terminologie. Comme ils l'expriment dans les focus groups, ils se reconnaissent plutôt comme des " parents avec une parentalité sans fin » figurant dans le titre de notre étude. En effet, selon eux, les offres actuelles pour les proches aidants se réfèrent à des situations d'accompagnement de personnes confrontées à des maladies chroniques et/ou des maladies neurodégénératives liées ou non au vieillissement. Pour eux, il n'existe actuellement pas d'offres analogues de soutiens spécifiques pour les parents accompagnant leur fils/fille en situation de handicap depuis la prime enfance. Dans les faits, ils nous indiquent s'orienter vers des dispositifs propres au domaine du handicap. Ils ont ainsi recours aux dispositifs offrant par exemple des séjours pendant les weekends, des vacances adaptées à la situation de leur fils/fille, ou des formations spécifiques aux problématiques auxquelles ils sont confrontés. Les parents estiment que les offres proposées par le domaine du handicap leur assurent une qualité de services adaptés à la situation de leur enfant. II semble que l'approche des parents envers ces dispositifs soit très différente de celle des proches aidants, qui eux cherchent du soutien pour les soulager dans leur implication liée à l'aide qu'ils apportent à leurs proches. Les parents de personnes en situation de handicap chercheraient quant à eux, au travers des dispositifs, à optimiser le bien-être de leur enfant, en lui permettant de participer à des activités qu'ils ne pourraient assurer. Ils estiment en outre que ces dispositifs leur offrent non pas une forme de répit pour eux-mêmes, mais un accès facilité à des espaces de vie et des loisirs adaptés pour leur enfant. Si les parents recourent aux services d'aide proposés par le domaine du handicap, ils reconnaissent que leurs besoins en termes de soulagement sont très largement sous-évalués, que ce soit par eux-mêmes ou par les professionnels de santé. Dans notre étude, nous avons proposé aux parents de réfléchir si un passage de relais du suivi de santé de leur enfant serait une forme possible de soulagement. Lors des échanges en groupe, ils nous ont indiqué pouvoir l'envisager dans certaines conditions. Ce, d'autant plus que leur sentiment de finitude les confronte à la nécessité de transmettre 
leur expertise à un ou des tiers afin de pérenniser le rôle de sentinelle de la santé de leur fils/fille.

Les parents nous indiquent ainsi pouvoir envisager de transmettre leur rôle de sentinelle de la santé à des équipes de professionnels à condition qu'elles soient qualifiées pour prendre ce relais. Cela nécessiterait selon eux, des professionnels formés à répondre aux problématiques de santé et disposant d'une connaissance plus globale qui comprendrait l'histoire de santé de leur fils/fille. Selon eux, ces professionnels pourraient alors devenir des interlocuteurs reconnus dans le réseau sanitaire et pourraient ainsi garantir la qualité et l'accessibilité aux soins pour leur proche. Les parents pourraient alors investir ces professionnels pour leur transmettre progressivement les aspects pratiques mentionnés plus haut (détection des problèmes de santé, appel et accompagnement chez le médecin, suivi de la prise en soin, etc.). Ils reconnaissent qu'ils seraient ainsi soulagés des contraintes inhérentes au suivi de santé de leur fils/fille et qu'ils pourraient gagner en sérénité dans la perspective de l'après eux. Cette approche d'empowerment (Campéon et Le Bihan-Youinou, 2016) offrirait selon nous aux parents le choix du moment et des modalités d'un passage de relais. Cette délégation choisie renforcerait leur sentiment de confiance et amoindrirait le sentiment de dépossession qu'ils expriment lorsqu'ils évoquent le passage de relais dans le système actuel. Cependant, les questions pragmatiques de cette transmission restent entières. Par exemple, à qui transmettre cette responsabilité du suivi de santé, comment la transmettre concrètement, et comment la faire valider légalement pour que la personne à qui cette responsabilité serait transmise soit reconnue par tous les acteurs (professionnels, institutions socio-éducatives et de santé, société civile) ? À l'occasion de la présentation des résultats de l'étude aux parentsparticipants, nous attendons à ce qu'ils puissent exprimer leurs points de vue sur les propositions de recommandations que l'équipe de recherche aura fait émerger compte tenu des analyses des données de l'étude, de la littérature et des politiques de santé publique actuelles.

\section{Aux confins de l'engagement des parents : réflexions et mises en œuvre à Genève}

Tout au long de leur vie, les parents ont fait face aux changements inhérents à l'avancée en âge de leur enfant. Au travers des problématiques de santé évolutives, des changements de lieu de vie, des nouveaux besoins liés à l'avancée en âge, les parents ont acquis un savoir qu'ils pourraient transmettre 
au réseau d'accompagnement de leur enfant adulte (McConnell et al., 2008). Cette transmission permettrait de soulager les parents vieillissants qui pourraient s'appuyer sur une forme de relais dans le système d'accompagnement. Le rôle central qu'ils occupent dans la coordination entre les différents professionnels qui interviennent dans la gestion de santé de leurs proches doit être selon nous considéré sous ses différents aspects. Ainsi, si la participation aux décisions de santé leur incombe légalement, ils pourraient être plus soutenus pour certains aspects pratiques tels que les prises de rendezvous, l'accompagnement et le transport aux consultations avec les praticiens de santé. Cette coopération nécessite une confiance mutuelle et une reconnaissance réciproque des expertises de chacun (Académie suisse des Sciences médicales, 2008). L'échange de savoirs entre les parents et les professionnels permettrait d'assurer un fil rouge et la continuité de la trajectoire d'accompagnement de la personne en situation de handicap tout au long de sa vie. Cette coopération est souvent précieuse à certaines étapes de vie, lors de l'intégration à l'école, des changements d'institution adaptée à l'âge et aux besoins, du passage du milieu familial au placement institutionnel (Dupuy-Vantroys, 2010). Les parents restent dépositaires, en tant que curateur, du suivi de santé de leur fils/fille arrivé à l'âge adulte et à une vie en institution. Cette responsabilité représente pour eux le dernier fil du lien qui les attache à leur enfant, fil auquel eux-mêmes peuvent se rattacher et qui permet de renforcer leur sentiment de parentalité sans fin. Ce sentiment est entretenu par la difficulté des professionnels socio-éducatifs à faire face aux problématiques de santé qui peuvent survenir chez les personnes qu'ils accompagnent, et ce d'autant plus lorsque celles-ci avancent en âge. Comme le souligne Laurent Decrop (2010, p. 38), "les équipes éducatives ont été formées à trouver le sens de leur travail dans la dynamique de la progression, de l'autonomisation et de l'acquisition de compétence. Le modèle intériorisé est celui de l'éducation d'un enfant en progrès. C'est ce modèle que viennent attaquer, l'avancée en âge, la maladie et la mort. " L'avancée en âge des personnes en situation de handicap questionne alors les professionnels et le système socio-éducatif et sanitaire sur les moyens à mettre œuvre pour assurer la qualité de leur accompagnement.

Dans les institutions du réseau genevois, actuellement la prise en charge des personnes adultes vieillissantes en situation de handicap est quasi exclusivement assurée dans des établissements socio-éducatifs spécialisés, du fait du nombre encore limité de résidents âgés. Toutefois, les institutions réfléchissent à la façon de faire face à la proportion croissante de cette population liée à l'augmentation de son espérance de vie. Pour cela, certaines 
institutions ont déjà mis en place des pôles seniors qui leur permettent d'accompagner leurs résidents jusqu'à la fin de leur vie. La création de ces unités spécialisées confronte les institutions au manque de professionnels qualifiés pour répondre aux besoins de santé propres à ces personnes vieillissantes et nécessite des investissements conséquents pour adapter leurs infrastructures. Face aux difficultés auxquelles les pôles seniors sont confrontés dans la gestion des situations de santé complexes de leurs aînés vulnérables, certaines institutions demandent aux parents de solliciter les établissements médico-sociaux pour personnes âgées pour accueillir leur proche. Bien que difficile à envisager et à réaliser pour les parents, certains relèvent que ce changement leur a apporté un réel soutien dans le suivi de santé et du bienêtre de leur fils/fille. En effet, les professionnels de santé de ces établissements disposent de compétences dans l'approche des troubles cognitifs et de réponses aux besoins spécifiques des personnes vieillissantes, transférables aux personnes en situation de handicap (gestion de la douleur, nutrition, soins palliatifs). De plus, ces établissements sont équipés d'infrastructures adaptées à la perte d'indépendance fonctionnelle des résidents (lit médicalisé, élévateur pour patient, sanitaires adaptés...) qui s'avèrent aussi facilitantes pour les personnes en situation de handicap.

\section{Perspectives et conclusion}

Dans les études que nous menons actuellement, nous nous intéressons aux influences du vieillissement des parents sur leur engagement dans le suivi de santé de leur fils/fille. Notre approche prend en compte le vieillissement conjoint des parents et de leur enfant et ce qu'il implique pour chacun des acteurs du réseau d'accompagnement. Explorer avec les parents curateurs, les possibilités d'un passage de relais de leur engagement dans le suivi de santé de leur fils-fille adulte touche, selon nous, au lien intime qui les attache à leur enfant. Pour approcher cette problématique sensible, nous avons développé une méthodologie de recherche où nous engageons les parents et les professionnels socio-éducatifs à l'expression et à la compréhension de leurs vécus. Ils sont ainsi co-constructeurs des propositions d'allégement de la charge que représente le suivi de santé de leur fils/fille qui prennent en compte leurs réalités multiples. La parole ainsi donnée aux parents et aux professionnels pourrait faciliter pour chacun la compréhension de leurs propres paradoxes. Celui vécu par les parents seniors à savoir, leur demande d'être soulagés et leur besoin de garder la maîtrise sur le suivi de santé de leur fils/fille. Celui vécu par les professionnels socio-éducatifs, qui tout en privilégiant une approche globale 
des personnes qu'ils accompagnent au quotidien, sont limités par leur défaut de qualification qui ne leur permet pas d'intégrer les problématiques de santé majorées par le vieillissement. Le constat actuel est que les solutions apportées relèvent du cas par cas selon les institutions. Une politique socio-sanitaire plus large permettrait d'harmoniser les réponses à ces situations par une reconnaissance de la nécessité de dotations en personnel qualifié et d'aménagements d'infrastructures adaptées. De plus, cela inciterait les institutions de formation socio-éducatives et de santé, à intégrer des enseignements sur la santé et le vieillissement des personnes en situation de handicap. Enfin, de développer les pratiques de collaboration interprofessionnelle dans le réseau socio-éducatif et sanitaire pour l'accompagnement, qui privilégie la perspective de la personne et de ses proches.

Apporter un soutien aux parents vieillissants qui leur permette de transmettre leur rôle de sentinelle de la santé aux professionnels serait une contribution à l'enjeu de santé communautaire posé par l'allongement de l'espérance de vie de tous. En conclusion, nous postulons que ce passage de relais entre parents et professionnels améliorerait la qualité de vie des parents et la qualité de travail des professionnels tout en garantissant la qualité du suivi de santé des personnes en situation de handicap.

\section{RÉFÉRENCES}

Académie suisse des Sciences médicales (2008). Traitement médical et prise en charge des personnes en situation de handicap. Directives et recommandations médico-éthiques. Berne, Suisse : Maison des Académies.

Art. 377-381, 398 du Code civil suisse du 10 décembre 1907, modification du 19 décembre 2008 (RO 2011 725).

Association francophone d'aide aux handicapés mentaux (2013). La Déficience intellectuelle, Fiche, Agence Wallonne Pour l'Intégration des personnes handicapées. Repéré à : https:// www.aviq.be/handicap/pdf/documentation/publications/informations_par ticulieres/fiches_deficiences_emploi/Fiche06-Deficienceintellectuelle.pdf

Azéma, B. et Martinez, N. (2005). Les personnes handicapées vieillissantes : espérances de vie et de santé ; qualité de vie. Revue française des affaires sociales, (2), 295-333. Repéré à : https://www.cairn.info/revue-francaisedes-affaires-sociales-2005-2-page-295.htm 
Breitenbach, N. (2003). Les aînés d'aujourd'hui et de demain, entre vieillesse et dépendance. Genève, Suisse : Insieme.

Berkman, B. (2006). Handbook of Social Work in Health and Aging. Oxford, Royaume-Uni : Oxford University Press.

Campéon, A. et Le Bihan-Youinou, B. (2016). Le développement des dispositifs d'aide aux aidants: Une démarche d'investissement social ? Informations sociales, 192(1), 88-97. Repéré à : https://www.cairn.info/revueinformations-sociales-2016-1-page-88.htm

Carlsson, E., Miniscalco, C., Kadesjö, B. et Laakso, K. (2016). Negotiating knowledge : Parents' experience of the neuropsychiatric diagnostic process for children with autism. International. Journal of Language \& Communication Disorders, 51(3), 328-338. doi:10.1111/1460-6984.12210

Cinter, F. et Paignon, A. (2019). Suivi de santé de personnes en situation de handicap avec une déficience intellectuelle : quelles réalités ? Revue suisse de pédagogie spécialisée, (2), 42-48. Repéré à : http://www.szhcsps.ch/r2019-06-05

Cuskelly, M. (2006). Parents of adults with an intellectual disability. Family Matters, (74), 20-26.

Decrop, L. (2010). Accompagner la fin de vie de personnes handicapées dans une association (Mémoire de Cafdes, École des Hautes Études en Santé Publique). Repéré à : http:// www.firah.org/centreressources/upload/notices3/2010/accompagner-la-fin-de-vie-depersonneshandicapees.pdf

Deschamps, J.-P. (2003). Une " relecture » de la charte d'Ottawa. Santé publique, 15(3), 313-325. Repéré à : https://www.cairn.info/revue-santepublique-2003-3-page-313.htm

Dupuy-Vantroys, A. (2010). Parents et professionnels partenaires dans l'accompagnement des personnes handicapées. Dans E. Hirsch (dir.), Traité de bioéthique. Tome 3. Handicaps, vulnérabilités, situations extrêmes (pp. 90-105). Toulouse, France : Erès. doi:10.3917/eres.

hirsc. 2010.03.0090

Gardou, C. (2016). Fondements et enjeux d'une société inclusive. Soins infirmiers, (5), 71-73.

Jecker-Parvex, M. et Breitenbach, N. (2012). Tracking an Elusive Population: Family Carers of Older Adults With Intellectual Disabilities in Romandy (Switzerland): Aging Family Carers in Romandy. Journal of Policy and Practice in Intellectual Disabilities, 9(3), 175-184. 
doi:10.1111/j.1741-1130.2012.00351.x

Jecker-Parvex, M., Charmillot, S., Thorin Burgdorfer, M., Devaux, A. et Korpès, J.-L. (2010). Vivre en famille pour les personnes vieillissantes ayant un handicap mental : quelles réalités ? quelles spécificités ? quel avenir? Synthèse de la recherche sur le handicap mental, I'habitat et le vieillissement, réalisée de décembre 2006 à juillet 2009 sur les cantons de Fribourg, Genève et Vaud. Insieme-Vaud. Repéré à : https://www.yumpu.com/fr/ document/read/3051208/vivre-en-famille-insieme-vaud

Korff-Sausse, S. (2007). L'impact du handicap sur les processus de parentalité. Reliance, 26(4), 22-29. Repéré à : https://www.cairn.info/revue-reliance2007-4-page-22.htm

Lalive d'Epinay Raemy, S. et Paignon, A. (2019). Providing equity of care for patients with intellectual and developmental disabilities in Western Switzerland: A descriptive intervention in a University Hospital. International Journal for Equity in Health, 18(1), 46. doi:10.1186/s12939-019-0948-8

Lamura, G., Döhner, H. et Kofahl, C. (2008). Family Carers of Older People in Europe: A Six-country Comparative Study. Münster, Allemagne: LIT Verlag.

Lindblad, B.-M., Rasmussen, B. H. et Sandman, P.-O. (2005). Being Invigorated in Parenthood: Parents' Experiences of Being Supported by Professionals When Having a Disabled Child. Journal of Pediatric Nursing, 20(4), 288-297. doi:10.1016/j.pedn.2005.04.015

McConnell, D., Matthews, J., Llewellyn, G., Mildon, R. et Hindmarsh, G. (2008). " Healthy Start ». A National Strategy for Parents with Intellectual Disabilities and Their Children. Journal of Policy and Practice in Intellectual Disabilities, 5(3), 194-202. doi:10.1111/ j.1741-1130.2008.00173.x

Office fédéral de la statistique. (2017). Enquête sur les revenus et les conditions de vie (SILC). Repéré à :

https://www.bfs.admin.ch/bfs/fr/home/statistiques/situation-economiquesociale-population/enquetes/silc.html

Office fédéral de la statistique. (2018). Enquête suisse sur la santé 2017 (n²131702). Repéré à https://www.bfs.admin.ch/bfs/fr/home/statistiques/catalogues-banquesdonnees/ publications.assetdetail.6426303.html

Ooi, K. L., Ong, Y. S., Jacob, S. A. et Khan, T. M. (2016). A meta-synthesis on parenting a child with autism. Neuropsychiatric Disease and Treatment, (12), 745-762. doi:10.2147/ NDT.S100634 
Organisation mondiale de la Santé. (2011). Rapport mondial sur le handicap. Repéré à : http://www.who.int/disabilities/world_report/2011/fr/

Piazza, V. E., Floyd, F. J., Mailick, M. R. et Greenberg, J. S. (2014). Coping and Psychological Health of Aging Parents of Adult Children with Developmental Disabilities. American Journal on Intellectual and Developmental Disabilities, 119(2), 186-198. doi:10.1352/ 1944-7558-119.2.186

Pozo, P. et Sarriá, E. (2015). Still stressed but feeling better: Well-being in autism spectrum disorder families as children become adults. Autism, 19(7), 805813. doi:10.1177/1362361315583191

Scelles, R. (2006). Devenir parent d'un enfant handicapé. Informations sociales, 132(4), 82-90. Repéré à : https://www.cairn.info/revue-informationssociales-2006-4-page-82. htm

Schneiderman, N., Ironson, G. et Siegel, S. D. (2005). Stress and Health: Psychological, Behavioral, and Biological Determinants. Annual Review of Clinical Psychology, (1), 607-628. doi:10.1146/annurev.clinpsy.1.102803.144141

Seltzer, M. M., Floyd, F. J., Song, J., Greenberg, J. S. et Hong, J. (2011). Midlife and Aging Parents of Adults with Intellectual and Developmental Disabilities: Impacts of Lifelong Parenting. American Journal on Intellectual and Developmental Disabilities, 116(6), 479-499. doi:10.1352/1944-7558116.6.479

Siu, Q. K. Y., Yi, H., Chan, R. C. H., Chio, F. H. N., Chan, D. F. Y. et Mak, W. W. S. (2018). The Role of Child Problem Behaviors in Autism Spectrum Symptoms and Parenting Stress: A Primary School-Based Study. Journal of Autism and Developmental Disorders, 9(3), 857-870. doi:10.1007/s10803-018-3791-7

Thomas, P., Hazif-Thomas, C. et Clément, J.-P. (2005). La plainte familiale dans la démence d'Alzheimer. NPG Neurologie - Psychiatrie - Gériatrie, 5(27), 3338.

doi:10.1016/S1627-4830(05)82577-2

Unapei. (2013). Livre Blanc : Pour une santé accessible aux personnes handicapées mentales. Repéré à : https://www.unapei.org/wpcontent/uploads/2018/11/livreblancfalc07_14.pdf

Waizbard-Bartov, E., Yehonatan-Schori, M. et Golan, O. (2019). Personal Growth Experiences of Parents to Children with Autism Spectrum Disorder. Journal of Autism and Developmental Disorders, 49(4), 1330-1341. doi:10.1007/s10803-018-3784-6 
Wicki, M. T. (2016). Withholding treatment and intellectual disability: Second survey on end-of-life decisions in Switzerland. SAGE Open Medicine, (4), 1-6. doi:10.1177/2050312116652637 